\title{
Synthesis, Characterization and Cytotoxicity Evaluation of Some Novel Pyridine Derivatives
}

\author{
Eman H. Tawfik, ${ }^{1}$ Khaled Samir Mohamed ${ }^{2, \star}$ Hemat M. Dardeer ${ }^{3}$ \\ and Ahmed Ali Fadda ${ }^{1}$ \\ ${ }^{1}$ Department of Chemistry, Faculty of Science, Mansoura University, ET-35516 Mansoura, Egypt \\ ${ }^{2}$ Engineering Chemistry Department, Higher Institute for Engineering and Technology, New Damietta, Egypt. \\ ${ }^{3}$ Chemistry Department, Faculty of Science, South Valley University, Qena 83523, Egypt \\ *Corresponding author: E-mail: Khaled_samirm@yahoo.com
}

Received: 28-02-2018

\begin{abstract}
Reaction of isonicotinaldehyde with 2-cyanoacetohydrazide afforded (E)-2-cyano- $N$ '-(pyridin-4-ylmethylene)acetohydrazide (1). Compound $\mathbf{1}$ was used as the precursor for the synthesis of novel pyridine derivatives by reaction with different arylidene malononitriles, malononitrile and acetylacetone to give pyridine derivatives $\mathbf{5 a - e ,} \mathbf{6}$ and 7, respectively. 4,4'-Bipyridine derivatives $9 \mathbf{9}-\mathbf{d}$ were synthesized by a three-component reaction of isonicotinaldehyde, 2-cyanoacetohydrazide and activated nitriles 8a-d. Treatment of compound $\mathbf{9 a}$ with different aromatic aldehydes gave $[1,2,4]$ triazolo[1,5-a]pyridine derivatives $\mathbf{1 1 a}-\mathbf{c}$. All reaction products were characterized by analytical and spectral data. For the novel compounds their bioactivity as antitumor agents was examined for in vitro cytotoxicity against HepG-2 and MCF-7. It was found that compounds $9 \mathbf{a}$ and $\mathbf{9 b}$ have high cytotoxic activity against both HepG-2 and MCF-7.
\end{abstract}

Keywords: Pyridine; 4,4'-bipyridine; isonicotinaldehyde; 2-cyanoacetohydrazide

\section{Introduction}

Among the important class of azaheterocycles, pyridine derivatives constitute one of the most significant classes of compounds as they broadly occur as vital struc-<smiles>COC(=O)NC(C(=O)N[C@@H](Cc1ccccc1)C(O)CNNC(=O)C(NC(C)=O)C(C)(C)C)C(C)(C)C</smiles>

Atazanavir

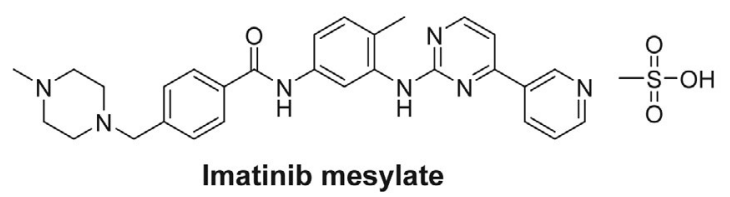

Figure 1. Chemical structures of atazanavir and imatinib mesylate tural subunits in many natural products, functional materials and pharmaceuticals ${ }^{1}$ that exhibit many motivating biological activities. ${ }^{2-4}$ For example atazanavir ${ }^{5}$ and imatinib mesylate ${ }^{6}$ (Figure 1) as two examples of drugs being prescribed for the treatment of HIV and chronic myelogenous leukemia, respectively.

Generally, pyridine derivatives have a huge spectrum of biological activities, like anti-leishmanial, ${ }^{7}$ anti-diabetic, ${ }^{8}$ anti-oxidant, ${ }^{9}$ antitumor ${ }^{10-12}$ and antiviral. ${ }^{13}$ Recently, some of pyridine derivatives were shown to act as potential targets for the development of new drugs for the treatment of cancer, ${ }^{14}$ as anti-platelet drugs, ${ }^{15}$ and antiproliferative agents. ${ }^{16}$

\section{Experimental}

\section{1. Materials and Methods}

\section{1. 1. Chemicals and Reagents}

All the chemicals and solvents used in this study were obtained from Merck (Germany). 


\section{1. 2. Instruments}

Melting points were recorded on Gallenkamp electric melting point apparatus (Electronic Melting Point Apparatus, Great Britain, London) and are uncorrected. Infrared spectra were recorded on Pye Unicam SP 1000 IR spectrophotometer (Thermoelectron Co. Egelsbach, Germany) using a $\mathrm{KBr}$ wafer technique. The ${ }^{1} \mathrm{H}$ NMR spectra were determined by a Bruker $400 \mathrm{MHz}$ spectrometer. DMSO- $d_{6}$ was used as the solvent, TMS was used as the internal standard and chemical shifts are given on $\delta$ scale in ppm. Mass spectra were determined on a GC-MS QP-100 EX Shimadzu (Japan). Microwave experiment was carried out using Milestone Start Microwave Lab Station. Elemental analyses were recorded on Perkin-Elmer 2400 Elemental analyzer at the Microanalytical Center at Cairo University, Cairo, Egypt.

\section{2. Synthesis}

Synthesis of (E)-2-Cyano- $N$-(pyridin-4-ylmethylene) acetohydrazide (1)

Method A: A mixture of isonicotinaldehyde $(1.07 \mathrm{~g}$, $0.01 \mathrm{~mol}), 2$-cyanoacetohydrazide $(9.9 \mathrm{~g}, 0.01 \mathrm{~mol})$ and TEA (2 drops) in THF or EtOH (15 mL) was refluxed for an appropriate time as shown in Table 1. The reaction progress was monitored by TLC. After completion of the reaction, the mixture was cooled to room temperature, the precipitate formed was collected by filtration and washed with ethyl acetate/petroleum ether (1:3), recrystallized from absolute ethanol to give pure compound $\mathbf{1}$.

Method B: A mixture of isonicotinaldehyde $(1.07 \mathrm{~g}$, $0.01 \mathrm{~mol})$ and 2-cyanoacetohydrazide $(9.9 \mathrm{~g}, 0.01 \mathrm{~mol})$ in a sealed tube was subjected to microwave irradiation at $700 \mathrm{~W}$ and microwave oven temperature $120^{\circ} \mathrm{C}$ for $20 \mathrm{sec}$. The reaction mixture was cooled to room temperature and washed by a mixture of ethyl acetate/petroleum ether (1:3). The precipitate formed was recrystallized from absolute ethanol to give the pure product $\mathbf{1}$ (yield 98\%).

Pale yellow crystals; yield: method A: $92 \%$ and $39 \%$ for THF and $\mathrm{EtOH}$, respectively; method B: 98\%; m.p. $169{ }^{\circ} \mathrm{C}$; IR (KBr): $v_{\max } 3235(\mathrm{NH}), 2259(\mathrm{CN}), 1704$ $(\mathrm{C}=\mathrm{O}) \mathrm{cm}^{-1} ;{ }^{1} \mathrm{H}$ NMR $\left(400 \mathrm{MHz}\right.$, DMSO- $\left.d_{6}\right): \delta 4.26(\mathrm{~s}$, $\left.2 \mathrm{H}, \mathrm{CH}_{2}\right), 8.02\left(\mathrm{~d}, J=7.2 \mathrm{~Hz}, 2 \mathrm{H}, \mathrm{C}_{3}-\mathrm{H}\right.$ and $\mathrm{C}_{5}-\mathrm{H}$ pyridine), $8.25(\mathrm{~s}, 1 \mathrm{H}, \mathrm{CH}=\mathrm{N}), 8.74\left(\mathrm{~d}, J=7.2 \mathrm{~Hz}, 2 \mathrm{H}, \mathrm{C}_{2}-\mathrm{H}\right.$ and $\mathrm{C}_{6}-\mathrm{H}$ pyridine), $11.86(\mathrm{~s}, 1 \mathrm{H}, \mathrm{NH}) ;{ }^{13} \mathrm{C}$ NMR $(100$ $\left.\mathrm{MHz}, \mathrm{DMSO}-d_{6}\right): \delta 25.02,119.39,122.87,141.01,145.72$, 149.61, 165.35; EI-MS: $m / z 188\left(\mathrm{M}^{+}, 10\right)$. Anal. Calcd. for $\mathrm{C}_{9} \mathrm{H}_{8} \mathrm{~N}_{4} \mathrm{O}(188.19)$ : C, 57.44; H, 4.29; N, 29.77. Found: C, $57.42 ; \mathrm{H}, 4.33 ; \mathrm{N}, 29.82$.

Synthesis of 2-Amino-cyano-6-oxo-4-aryl-1-((pyridin4-ylmethylene)amino))-1,6-dihydropyridine-3,5-dicarbonitriles $5 \mathbf{a}-\mathbf{e}$

General procedure: To a solution of compound $\mathbf{1}$ $(1.88 \mathrm{~g}, 0.01 \mathrm{~mol})$ in $\mathrm{EtOH}(20 \mathrm{~mL})$, arylidine malononitriles $2 \mathbf{a}-\mathbf{e}(0.01 \mathrm{~mol})$ and a catalytic amount of trimethylamine were added. The reaction mixture was heated under reflux for 17-20 h (TLC controlled), then, the reaction mixture was left to cool. The precipitate that formed was filtered off, washed with ethyl acetate and recrystallized from ethanol to give the products $\mathbf{5 a}-\mathbf{e}$.

2-Amino-6-oxo-4-phenyl-1-((pyridin-4-ylmethylene) amino)-1,6-dihydropyridine-3,5-dicarbonitrile (5a)

Brown crystals; yield: $20 \%$; m.p. $>300{ }^{\circ} \mathrm{C}$; IR $(\mathrm{KBr})$ : $v_{\max } 3345,3390\left(\mathrm{NH}_{2}\right), 2214,2206(2 \times \mathrm{CN}), 1673(\mathrm{C}=\mathrm{O})$ $\mathrm{cm}^{-1} ;{ }^{1} \mathrm{H}$ NMR (400 MHz, DMSO- $\left.d_{6}\right): \delta 6.55\left(\mathrm{~s}, 2 \mathrm{H}, \mathrm{NH}_{2}\right)$, 7.10-7.50 (m, 5H, Ar-H), $7.99\left(\mathrm{~d}, J=7.2 \mathrm{~Hz}, 2 \mathrm{H}, \mathrm{C}_{3}-\mathrm{H}\right.$ and $\mathrm{C}_{5}-\mathrm{H}$ pyridine), $8.43(\mathrm{~s}, 1 \mathrm{H}, \mathrm{CH}=\mathrm{N}), 8.73(\mathrm{~d}, J=7.2 \mathrm{~Hz}$, $2 \mathrm{H}, \mathrm{C}_{2}-\mathrm{H}$ and $\mathrm{C}_{6}-\mathrm{H}$ pyridine); ${ }^{13} \mathrm{C}$ NMR $(100 \mathrm{MHz}, \mathrm{DM}-$ SO- $\left.d_{6}\right): \delta 77.83,115.23,115.81,116.29,122.74,126.90$, $128.76,129.20,134.88,145.83,149.89,152.66,158.90$, 162.34, 168.88; EI-MS: $m / z 340\left(\mathrm{M}^{+}, 50 \%\right)$. Anal. Calcd. for $\mathrm{C}_{19} \mathrm{H}_{12} \mathrm{~N}_{6} \mathrm{O}$ (340.35): C, 67.05; H, 3.55; N, 24.69. Found: C, 67.12; H, 3.49; N, 24.72.

2-Amino-4-(4-methoxyphenyl)-6-oxo-1-((pyridin-4-ylmethylene)amino)-1,6-dihydropyridine-3,5-dicarbonitrile (5b)

Dark brown crystals; yield: $31 \%$; m.p. $208{ }^{\circ} \mathrm{C}$. IR $(\mathrm{KBr}): v_{\max } 3320,3385\left(\mathrm{NH}_{2}\right), 2212,2235(2 \times \mathrm{CN}), 1669$ $(\mathrm{C}=\mathrm{O}) \mathrm{cm}^{-1} ;{ }^{1} \mathrm{H}$ NMR $\left(400 \mathrm{MHz}, \mathrm{DMSO}-d_{6}\right): \delta 3.85(\mathrm{~s}$, $\left.3 \mathrm{H}, \mathrm{CH}_{3}\right), 6.58\left(\mathrm{~s}, 2 \mathrm{H}, \mathrm{NH}_{2}\right), 6.95(\mathrm{~d}, J=8.0 \mathrm{~Hz}, 2 \mathrm{H}, \mathrm{Ar}-$ $\mathrm{H}), 7.62(\mathrm{~d}, J=8.1 \mathrm{~Hz}, 2 \mathrm{H}, \mathrm{Ar}-\mathrm{H}), 8.04(\mathrm{~d}, J=7.2 \mathrm{~Hz}, 2 \mathrm{H}$, $\mathrm{C}_{3}-\mathrm{H}$ and $\mathrm{C}_{5}-\mathrm{H}$ pyridine $), 8.43(\mathrm{~s}, 1 \mathrm{H}, \mathrm{CH}=\mathrm{N}), 8.72(\mathrm{~d}, \mathrm{~J}=$ $7.2 \mathrm{~Hz}, 2 \mathrm{H}, \mathrm{C}_{2}-\mathrm{H}$ and $\mathrm{C}_{6}-\mathrm{H}$ pyridine); ${ }^{13} \mathrm{C}$ NMR $(100$ MHz, DMSO- $\left.d_{6}\right): \delta 59.24,77.64,115.11,115.83,116.22$, $116.79,122.58,125.69,131.94,146.04,149.91,152.75$, 158.14, 158.99, 161.67, 169.52; EI-MS: $m / z 370\left(\mathrm{M}^{+}, 18 \%\right)$. Anal. Calcd. for $\mathrm{C}_{20} \mathrm{H}_{14} \mathrm{~N}_{6} \mathrm{O}_{2}$ (370.37): C, 64.86; $\mathrm{H}, 3.81 ; \mathrm{N}$, 22.69. Found: C, 64.84; H, 3.79; N, 22.63.

2-Amino-4-(4-hydroxyphenyl)-6-oxo-1-((pyridin-4-ylmethylene)amino)-1,6-dihydropyridine-3,5-dicarbonitrile $(5 c)$

Brown crystals; yield: $33 \%$; m.p. $214{ }^{\circ} \mathrm{C}$; IR $(\mathrm{KBr})$; $v_{\max } 3425(\mathrm{OH}), 3355,3314\left(\mathrm{NH}_{2}\right), 2213,2229(2 \times \mathrm{CN})$, $1679(\mathrm{C}=\mathrm{O}) \mathrm{cm}^{-1} ;{ }^{1} \mathrm{H}$ NMR $\left(400 \mathrm{MHz}, \mathrm{DMSO}-d_{6}\right): \delta 6.58$ $\left(\mathrm{s}, 2 \mathrm{H}, \mathrm{NH}_{2}\right), 6.74(\mathrm{~d}, J=7.8 \mathrm{~Hz}, 2 \mathrm{H}, \mathrm{Ar}-\mathrm{H}), 7.44(\mathrm{~d}, J=7.8$ $\mathrm{Hz}, 2 \mathrm{H}, \mathrm{Ar}-\mathrm{H}), 8.00\left(\mathrm{~d}, J=7.2 \mathrm{~Hz}, 2 \mathrm{H}, \mathrm{C}_{3}-\mathrm{H}\right.$ and $\mathrm{C}_{5}-\mathrm{H}$ pyridine), $8.40(\mathrm{~s}, 1 \mathrm{H}, \mathrm{CH}=\mathrm{N}), 8.74(\mathrm{~d}, J=7.2 \mathrm{~Hz}, 2 \mathrm{H}$, $\mathrm{C}_{2}-\mathrm{H}$ and $\mathrm{C}_{6}-\mathrm{H}$ pyridine), $9.77(\mathrm{~s}, 1 \mathrm{H}, \mathrm{OH}) ;{ }^{13} \mathrm{C} \mathrm{NMR}(100$ $\left.\mathrm{MHz}, \mathrm{DMSO}-d_{6}\right): \delta 77.37,115.23,115.88,116.17,116.89$, $122.75,126.73,130.88,145.83,149.93,152.82,158.12$, 159.18, 161.73, 169.43; EI-MS: $m / z 356\left(\mathrm{M}^{+}, 9 \%\right)$. Anal. Calcd. for $\mathrm{C}_{19} \mathrm{H}_{12} \mathrm{~N}_{6} \mathrm{O}_{2}(356.34)$ : C, 64.04; H, 3.39; N, 23.58. Found: C, 63.99; H, 3.42; N, 23.51.

2-Amino-4-(4-(dimethylamino)phenyl)-6-oxo-1-((pyridin-4-ylmethylene)amino)-1,6-dihydropyridine3,5-dicarbonitrile (5d)

Brown crystals; yield: $51 \%$; m.p. $220{ }^{\circ} \mathrm{C}$; IR $(\mathrm{KBr})$ :

$v_{\max } 3397,3381\left(\mathrm{NH}_{2}\right), 2224,2241(2 \times \mathrm{CN}), 1670(\mathrm{C}=\mathrm{O})$ 
$\mathrm{cm}^{-1} ;{ }^{1} \mathrm{H}$ NMR $\left(400 \mathrm{MHz}, \mathrm{DMSO}-d_{6}\right): \delta 3.13\left(\mathrm{~s}, 6 \mathrm{H}, \mathrm{CH}_{3}\right)$, $6.58\left(\mathrm{~s}, 2 \mathrm{H}, \mathrm{NH}_{2}\right), 6.81$ (d, J = 8.2 Hz, 2H, Ar-H), 7.23 (d, $J$ $=8.2 \mathrm{~Hz}, 2 \mathrm{H}, \mathrm{Ar}-\mathrm{H}), 8.00\left(\mathrm{~d}, J=7.2 \mathrm{~Hz}, 2 \mathrm{H}, \mathrm{C}_{3}-\mathrm{H}\right.$ and $\mathrm{C}_{5}-\mathrm{H}$ pyridine), $8.42(\mathrm{~s}, 1 \mathrm{H}, \mathrm{CH}=\mathrm{N}), 8.72(\mathrm{~d}, J=7.3 \mathrm{~Hz}$, $2 \mathrm{H}, \mathrm{C}_{2}-\mathrm{H}$ and $\mathrm{C}_{6}-\mathrm{H}$ pyridine); ${ }^{13} \mathrm{C}$ NMR (100 MHz, DMSO- $\left.d_{6}\right): \delta 40.62,77.06,112.89,115.61,115.97,116.22$, $122.57,123.16,131.11,146.34,150.04,150.96,153.08$, 158.97, 161.82, 169.07; EI-MS: $m / z 383$ (M+, 94\%). Anal. Calcd. for $\mathrm{C}_{21} \mathrm{H}_{17} \mathrm{~N}_{7} \mathrm{O}$ (383.42): C, 65.79; H, 4.47; N, 25.57 . Found: C, 65.71; H, 4.51; N, 25.60.

2-Amino-4-(4-hydroxy-3-methoxyphenyl)-6-oxo-1((pyridin-4-ylmethylene) amino)-1,6-dihydropyridine-3,5-dicarbonitrile (5e)

Orang red crystals; yield: $22 \%$; m.p. $240{ }^{\circ} \mathrm{C}$. IR $(\mathrm{KBr}): v_{\max } 3418(\mathrm{OH}), 3337,3308\left(\mathrm{NH}_{2}\right), 2207,2225$ $(2 \times \mathrm{CN}), 1666(\mathrm{C}=\mathrm{O}) \mathrm{cm}^{-1} ;{ }^{1} \mathrm{H}$ NMR $(400 \mathrm{MHz}, \mathrm{DM}-$ $\left.\mathrm{SO}-d_{6}\right): \delta 4.11\left(\mathrm{~s}, 3 \mathrm{H}, \mathrm{OCH}_{3}\right), 6.53\left(\mathrm{~s}, 2 \mathrm{H}, \mathrm{NH}_{2}\right), 6.78-7.12$ $(\mathrm{m}, 3 \mathrm{H}, \mathrm{Ar}-\mathrm{H}), 8.01\left(\mathrm{~d}, J=7.2 \mathrm{~Hz}, 2 \mathrm{H}, \mathrm{C}_{3}-\mathrm{H}\right.$ and $\mathrm{C}_{5}-\mathrm{H}$ pyridine), $8.42(\mathrm{~s}, 1 \mathrm{H}, \mathrm{CH}=\mathrm{N}), 8.75(\mathrm{~d}, J=7.2 \mathrm{~Hz}, 2 \mathrm{H}$, $\mathrm{C}_{2}-\mathrm{H}$ and $\mathrm{C}_{6}-\mathrm{H}$ pyridine), $9.57(\mathrm{~s}, 1 \mathrm{H}, \mathrm{OH}) ;{ }^{13} \mathrm{C}$ NMR $\left(100 \mathrm{MHz}, \mathrm{DMSO}-d_{6}\right): \delta 58.38,77.13,114.92,115.44$, $115.87,116.89,117.35,122.92,123.79,127.14,144.91$, $148.49,149.72,150.98,153.42,158.24,161.62,169.38$; EIMS: $m / z 386\left(\mathrm{M}^{+}, 31 \%\right)$. Anal. Calcd. for $\mathrm{C}_{20} \mathrm{H}_{14} \mathrm{~N}_{6} \mathrm{O}_{3}$ (386.37): C, 62.17; H, 3.65; N, 21.75. Found: C, 62.21; H, $3.58 ; \mathrm{N}, 21.70$.

Synthesis of 4,6-Diamino-2-oxo-1-((pyridin-4-ylmethylene)amino)-1,2-dihydropyridine-3-carbonitrile (6)

A mixture of compound 1 (1.88 g, $0.005 \mathrm{~mol})$ and malononitrile $(0.01 \mathrm{~mol})$ in $20 \mathrm{~mL}$ of absolute EtOH containing 3 drops of triethylamine was refluxed for $9 \mathrm{~h}$ (TLC controlled). Then, the reaction mixture was left to cool and the precipitated solid was filtered off, dried, washed with ethyl acetate and recrystallized from absolute $\mathrm{EtOH}$ to afford compound $\mathbf{6}$.

Yellow crystals; yield: $65 \%$; m.p. $>300{ }^{\circ} \mathrm{C}$; IR $(\mathrm{KBr})$ : $v_{\max } 3399,3388,3347,3321\left(2 \times \mathrm{NH}_{2}\right), 2216(\mathrm{CN}), 1683$ $(\mathrm{C}=\mathrm{O}) \mathrm{cm}^{-1} ;{ }^{1} \mathrm{H}$ NMR $\left(400 \mathrm{MHz}, \mathrm{DMSO}-d_{6}\right): \delta 4.62(\mathrm{~s}$, $1 \mathrm{H}, \mathrm{CH}), 4.65$ (s, 2H, $\left.\mathrm{NH}_{2}\right), 6.68\left(\mathrm{~s}, 2 \mathrm{H}, \mathrm{NH}_{2}\right), 8.03$ (d, $J=$ $7.2 \mathrm{~Hz}, 2 \mathrm{H}, \mathrm{C}_{3}-\mathrm{H}, \mathrm{C}_{5}-\mathrm{H}$ pyridine), $8.43(\mathrm{~s}, 1 \mathrm{H}, \mathrm{CH}=\mathrm{N})$, $8.71\left(\mathrm{~d}, J=7.2 \mathrm{~Hz}, 2 \mathrm{H}, \mathrm{C} 2-\mathrm{H}, \mathrm{C} 6-\mathrm{H}\right.$ pyridine); ${ }^{13} \mathrm{C} \mathrm{NMR}$ $\left(100 \mathrm{MHz}, \mathrm{DMSO}-d_{6}\right): \delta 72.83,86.27,116.17,123.55$, $143.94,146.25,151.47,155.18,163.48,178.28$; EI-MS: $m / z$ $254\left(\mathrm{M}^{+}, 100 \%\right)$. Anal. Calcd. for $\mathrm{C}_{12} \mathrm{H}_{10} \mathrm{~N}_{6} \mathrm{O}$ (254.25): C, 56.69; H, 3.96; N, 33.05. Found: C, 56.77; H, 4.01; N, 32.96.

Synthesis of 4,6-Dimethyl-2-oxo-1-((pyridin-4-ylmethylene)amino)-1,2-dihydropyridine-3-carbonitrile (7)

To a solution of $1(1.88 \mathrm{~g}, 0.01 \mathrm{~mol})$ and acetylacetone $(1.001 \mathrm{~g}, 0.01 \mathrm{~mol})$ in $20 \mathrm{~mL}$ of absolute $\mathrm{EtOH}$ containing a few drops of trimethylamine were added. The reaction mixture was heated under reflux for $18 \mathrm{~h}$. After the completion of the reaction, the reaction mixture was cooled and the separated solid product was collected by filtration, washed with ethanol, dried, and recrystallized from $\mathrm{EtOH}$ to give compound 7.

Buff crystals; yield: $61 \%$; m.p $190{ }^{\circ} \mathrm{C}$; IR (KBr): $v_{\max }$ $2208(\mathrm{CN}), 1674(\mathrm{C}=\mathrm{O}) \mathrm{cm}^{-1} ;{ }^{1} \mathrm{H}$ NMR (400 MHz, DM$\left.\mathrm{SO}-d_{6}\right): \delta 2.13\left(\mathrm{~s}, 3 \mathrm{H}, \mathrm{CH}_{3}\right), 2.22\left(\mathrm{~s}, 3 \mathrm{H}, \mathrm{CH}_{3}\right), 5.66(\mathrm{~s}, 1 \mathrm{H}$, $\mathrm{CH}), 8.01$ (d, $J=7.2 \mathrm{~Hz}, 2 \mathrm{H}, \mathrm{C}_{3}-\mathrm{H}, \mathrm{C}_{5}-\mathrm{H}$ pyridine), 8.41 (s, $1 \mathrm{H}, \mathrm{CH}=\mathrm{N}), 8.70\left(\mathrm{~d}, J=7.2 \mathrm{~Hz}, 2 \mathrm{H}, \mathrm{C}_{2}-\mathrm{H}, \mathrm{C}_{6}-\mathrm{H}\right.$ pyridine); ${ }^{13} \mathrm{C}$ NMR (100 MHz, DMSO- $\left.d_{6}\right): \delta 18.24,22.03,110.94$, $116.83,118.07,122.44,135.88,145.57,150.21,153.47$, 155.26, 161.47; EI-MS: $m / z 252\left(\mathrm{M}^{+}, 100 \%\right)$. Anal. Calcd. for $\mathrm{C}_{14} \mathrm{H}_{12} \mathrm{~N}_{4} \mathrm{O}$ (252.28): C, 66.65; H, 4.79; N, 22.21. Found: C, 66.49; H, 4.81; N, 22.29.

Synthesis of 1,6-Diamino-2-oxo-5-(alkyl)-1,2-dihydro[4,4'-bipyridine]-3-carbonitriles $9 a-d$

General procedure: To a mixture of isonicotinaldehyde $(1.07 \mathrm{~g}, 0.01 \mathrm{~mol})$, activated nitriles $8 \mathbf{a}-\mathbf{d}(0.01 \mathrm{~mol})$ and 2-cyanoacetohydrazide $(0.99 \mathrm{~g}, 0.01 \mathrm{~mol})$ absolute EtOH $(20 \mathrm{~mL})$ containing three drops of piperidine was added. The reaction mixture was heated under reflux for 6-8 h (TLC controlled). The reaction mixture was left to cool to the room temperature, then the solid formed was filtered off and recrystallized from absolute $\mathrm{EtOH}$ to give compounds $9 a-d$

\section{1,6-Diamino-2-oxo-1,2-dihydro-[4,4'-bipyridine]-3,5- dicarbonitrile (9a)}

Brown crystals; yield: 71\%; m.p. $>300{ }^{\circ} \mathrm{C}$; IR (KBr): $v_{\max } 3428,3373,3366,3345\left(2 \times \mathrm{NH}_{2}\right), 2202,2180(2 \mathrm{CN})$, $1665(\mathrm{C}=\mathrm{O}) \mathrm{cm}^{-1} ;{ }^{1} \mathrm{H}$ NMR (400 MHz, DMSO- $\left.d_{6}\right): \delta 5.22$ (s, $\left.2 \mathrm{H}, \mathrm{NH}_{2}\right), 6.56\left(\mathrm{~s}, 2 \mathrm{H}, \mathrm{NH}_{2}\right), 7.51(\mathrm{~d}, J=7.2 \mathrm{~Hz}, 2 \mathrm{H}$, $\mathrm{C}_{3}-\mathrm{H}, \mathrm{C}_{5}-\mathrm{H}$ pyridine), $8.63\left(\mathrm{~d}, J=7.2 \mathrm{~Hz}, 2 \mathrm{H}, \mathrm{C}_{2}-\mathrm{H}, \mathrm{C}_{6}-\mathrm{H}\right.$ pyridine); ${ }^{13} \mathrm{C}$ NMR (100 MHz, DMSO- $\left.d_{6}\right): \delta 77.42$, $115.24,116.27,122.05,122.84,145.14,150.44,159.11$, 161.57, 171.34; EI-MS: $m / z 186\left(\mathrm{M}^{+}, 100 \%\right)$. Anal. Calcd. for $\mathrm{C}_{12} \mathrm{H}_{8} \mathrm{~N}_{6} \mathrm{O}(252.24)$ : C, 57.14; H, 3.20; N, 33.32. Found: C, 57.21; H, 3.16; N, 33.33.

1,6-Diamino-2-oxo-5-(phenylsulfonyl)-1,2-dihydro[4,4'-bipyridine]-3-carbonitrile (9b)

Orange crystals; yield: 53\%; m.p. $145{ }^{\circ} \mathrm{C}$; IR (KBr): $v_{\max } 3447,3403,3334,3070\left(2 \times \mathrm{NH}_{2}\right), 2202(\mathrm{CN}), 1669$ $(\mathrm{C}=\mathrm{O}) \mathrm{cm}^{-1} ;{ }^{1} \mathrm{H}$ NMR $\left(400 \mathrm{MHz}, \mathrm{DMSO}-d_{6}\right): \delta 5.48(\mathrm{~s}$, $\left.2 \mathrm{H}, \mathrm{NH}_{2}\right), 6.57$ (s, 2H, $\left.\mathrm{NH}_{2}\right), 7.50-7.80(\mathrm{~m}, 7 \mathrm{H}, \mathrm{Ar}-\mathrm{H}$ and $\mathrm{C}_{3}-\mathrm{H}, \mathrm{C}_{5}-\mathrm{H}$ pyridine), 8.59 (d, $J=7.2 \mathrm{~Hz}, 2 \mathrm{H}, \mathrm{C}_{2}-\mathrm{H}, \mathrm{C}_{6}-\mathrm{H}$ pyridine); ${ }^{13} \mathrm{C}$ NMR (100 MHz, DMSO- $\left.d_{6}\right): \delta 102.45$, $116.49,121.69,122.82,128.95,130.14,133.18,141.89$, $143.15,143.83,151.26,161.77,170.89$; EI-MS: $m / z 367$ $\left(\mathrm{M}^{+}, 8 \%\right)$. Anal. Calcd. for $\mathrm{C}_{17} \mathrm{H}_{13} \mathrm{~N}_{5} \mathrm{O}_{3} \mathrm{~S}$ (367.38): C, 55.58; H, 3.57; N, 19.06; S, 8.73. Found: C, 55.58; H, 3.57; $\mathrm{N}, 19.06 ; \mathrm{S}, 8.73$.

Ethyl 1,2-Diamino-5-cyano-6-oxo-1,6-dihydro-[4,4'-bipyridine]-3-carboxylate (9c)

Yellow crystals; yield: $48 \%$; m.p. $180-190{ }^{\circ} \mathrm{C}$; IR $(\mathrm{KBr}): v_{\max } 3351,3339,3335,3305\left(2 \times \mathrm{NH}_{2}\right), 2215(\mathrm{CN})$, 
1725, $1674(2 \times \mathrm{C}=\mathrm{O}) \mathrm{cm}^{-1} ;{ }^{1} \mathrm{H}$ NMR $(400 \mathrm{MHz}, \mathrm{DM}-$ SO- $\left.d_{6}\right): \delta 1.01\left(\mathrm{t}, J=7.2 \mathrm{~Hz} 3 \mathrm{H}, \mathrm{CH}_{3}\right), 4.03(\mathrm{q}, J=6.8 \mathrm{~Hz}$ $\left.2 \mathrm{H}, \mathrm{CH}_{2}\right), 5.39\left(\mathrm{~s}, 2 \mathrm{H}, \mathrm{NH}_{2}\right), 7.08\left(\mathrm{~s}, 2 \mathrm{H}, \mathrm{NH}_{2}\right), 7.56(\mathrm{~d}, J=$ $7.4 \mathrm{~Hz}, 2 \mathrm{H}, \mathrm{C}_{3}-\mathrm{H}, \mathrm{C}_{5}-\mathrm{H}$ pyridine), $8.62(\mathrm{~d}, J=7.4 \mathrm{~Hz}, 2 \mathrm{H}$, $\mathrm{C}_{2}-\mathrm{H}, \mathrm{C}_{6}-\mathrm{H}$ pyridine); ${ }^{13} \mathrm{C}$ NMR (100 MHz, DMSO- $\left.d_{6}\right): \delta$ 15.37, 64.49, 99.56, 116.47, 121.82, 122.43, 143.73, 150.13, 152.85, 161.04, 167.28, 171.11; EI-MS: $m / z 299\left(\mathrm{M}^{+}\right.$, $100 \%)$. Anal. Calcd. for $\mathrm{C}_{14} \mathrm{H}_{13} \mathrm{~N}_{5} \mathrm{O}_{3}(299.29)$ : C, 56.18; $\mathrm{H}$, 4.38 ; N, 23.40. Found: C, 56.21; H, 4.33; N, 23.41.

1,6-Diamino-5-(benzo[d] thiazol-2-yl)-2-oxo-1,2-dihydro-[4,4'-bipyridine]-3-carbonitrile (9d)

Yellow crystals; yield: $73 \%$; m.p. $315{ }^{\circ} \mathrm{C}$; IR (KBr): $v_{\max } 3400,3391,3255,3066\left(2 \times \mathrm{NH}_{2}\right), 2209(\mathrm{CN}), 1661$ $(\mathrm{C}=\mathrm{O}) \mathrm{cm}^{-1}$; ${ }^{1} \mathrm{H}$ NMR $\left(400 \mathrm{MHz}, \mathrm{DMSO}-d_{6}\right): \delta 5.36(\mathrm{~s}$, $\left.2 \mathrm{H}, \mathrm{NH}_{2}\right), 7.08\left(\mathrm{~s}, 2 \mathrm{H}, \mathrm{NH}_{2}\right), 7.50-8.00(\mathrm{~m}, 7 \mathrm{H}, \mathrm{Ar}-\mathrm{H})$, $8.71\left(\mathrm{~d}, J=7.2 \mathrm{~Hz}, 2 \mathrm{H}, \mathrm{C}_{2}-\mathrm{H}, \mathrm{C}_{6}-\mathrm{H}\right.$ pyridine); ${ }^{13} \mathrm{C} \mathrm{NMR}$ $\left(100 \mathrm{MHz}, \mathrm{DMSO}-d_{6}\right): \delta 102.14,116.40,121.23,121.98$, $122.73,124.85,125.94,126.48,138.38,141.29,145.34$, 150.77, 155.26, 161.14, 161.99, 171.57; EI-MS: $m / z 360$ $\left(\mathrm{M}^{+}, 83 \%\right)$. Anal. Calcd. for $\mathrm{C}_{18} \mathrm{H}_{12} \mathrm{~N}_{6} \mathrm{OS}$ (360.40): C, 59.99; H, 3.36; N, 23.32; S, 8.90. Found: C, 59.95; H, 3.41; N, 23.34; S, 8.87.

Synthesis of 5-Oxo-2-aryl-7-(pyridin-4-yl)-1,2,3,5-tetrahydro- $[1,2,4]$ triazolo $[1,5-a]$ pyridine-6,8-dicarbonitriles $11 \mathrm{a}-\mathrm{c}$

General procedure:A solution of compound 9a (2.52 $\mathrm{g}, 0.01 \mathrm{~mol})$, aromatic aldehydes $\mathbf{1 0 a}-\mathbf{c}(0.01 \mathrm{~mol})$ in 1,4 dioxane and/or DMF $(25 \mathrm{~mL})$ containing a catalytic amount of piperidine ( 3 drops) was heated under reflux for $12-15 \mathrm{~h}$. The reaction was monitored by TLC. The product that was precipitated on cooling to room temperature was filtered off and recrystallized from absolute $\mathrm{EtOH}$ to give the compounds $11 \mathbf{a}-\mathbf{c}$.

5-Oxo-2-phenyl-7-(pyridin-4-yl)-1,2,3,5-tetrahydro$[1,2,4]$ triazolo[1,5-a]pyridine-6,8-dicarbonitrile (11a)

Brown crystals; yield: $65 \%$; m.p. $>300^{\circ} \mathrm{C}$; IR $(\mathrm{KBr})$ : $v_{\max } 3245,3283(2 \times \mathrm{NH}), 2216,2195(2 \times \mathrm{CN}), 1679(\mathrm{C}=\mathrm{O})$ $\mathrm{cm}^{-1} ;{ }^{1} \mathrm{H}$ NMR $\left(400 \mathrm{MHz}, \mathrm{DMSO}-d_{6}\right): \delta 4.81(\mathrm{~s}, 1 \mathrm{H}, \mathrm{NH})$, $5.11(\mathrm{~s}, 1 \mathrm{H}, \mathrm{CH}), 7.21-7.59\left(\mathrm{~m}, 8 \mathrm{H}, \mathrm{Ar}-\mathrm{H}, \mathrm{NH}\right.$ and $\mathrm{C}_{3}-\mathrm{H}$, $\mathrm{C}_{5}-\mathrm{H}$ pyridine), $8.66\left(\mathrm{~d}, J=7.2 \mathrm{~Hz}, 2 \mathrm{H}, \mathrm{C}_{2}-\mathrm{H}, \mathrm{C}_{6}-\mathrm{H}\right.$ pyridine); ${ }^{13} \mathrm{C}$ NMR (100 MHz, DMSO- $\left.d_{6}\right): \delta 87.47,98.79$, $116.78,117.14,122.16,123.48,126.77,127.14,130.47$, 143.59, 146.41, 150.72, 160.62, 162.34, 170.83; EI-MS: $\mathrm{m} / z$ $340\left(\mathrm{M}^{+}, 19 \%\right)$. Anal. Calcd. for $\mathrm{C}_{19} \mathrm{H}_{12} \mathrm{~N}_{6} \mathrm{O}$ (340.35): C, 67.05; H, 3.55; N, 24.69. Found: C, 67.11; H, 3.58; N, 24.60 .

2-(4-Methoxyphenyl)-5-oxo-7-(pyridin-4-yl)-1,2,3,5tetrahydro- $[1,2,4]$ triazolo $[1,5-a]$ pyridine-6,8-dicarbonitrile (11b)

Brown crystals; yield: $63 \%$; m.p. $>300{ }^{\circ} \mathrm{C}$; IR $(\mathrm{KBr})$ :

$v_{\max } 3243,3291(2 \times \mathrm{NH}), 2215,2195(2 \times \mathrm{CN}), 1678(\mathrm{C}=\mathrm{O})$

$\mathrm{cm}^{-1} ;{ }^{1} \mathrm{H}$ NMR (400 MHz, DMSO- $d_{6}$ ): $\delta 4.05$ (s, $3 \mathrm{H}$,
$\left.\mathrm{OCH}_{3}\right), 4.77(\mathrm{~s}, 1 \mathrm{H}, \mathrm{NH}), 5.08(\mathrm{~s}, 1 \mathrm{H}, \mathrm{CH}), 6.95(\mathrm{~d}, J=8.4$ $\mathrm{Hz}, 2 \mathrm{H}, \mathrm{Ar}-\mathrm{H}), 7.29$ (s, $1 \mathrm{H}, \mathrm{NH}), 7.40-7.60$ (m, 5H, Ar-H, $\mathrm{NH}$ and $\mathrm{C}_{3}-\mathrm{H}, \mathrm{C}_{5}-\mathrm{H}$ pyridine $), 8.66\left(\mathrm{~d}, J=7.2 \mathrm{~Hz}, 2 \mathrm{H}, \mathrm{C}_{2^{-}}\right.$ $\mathrm{H}, \mathrm{C}_{6}-\mathrm{H}$ pyridine); ${ }^{13} \mathrm{C}$ NMR (100 MHz, DMSO- $\left.d_{6}\right): \delta$ 59.74, 87.51, 98.78, 115.78, 116.78, 117.09, 122.24, 123.50, $129.03,138.14,144.10,150.84,159.25,160.81,162.27$, 171.09; EI-MS: $m / z 370\left(\mathrm{M}^{+}, 30 \%\right)$. Anal. Calcd. for $\mathrm{C}_{20} \mathrm{H}-$ ${ }_{14} \mathrm{~N}_{6} \mathrm{O}_{2}$ (370.37): C, 64.86; H, 3.81; N, 22.69. Found: C, 64.91; H, 3.75; N, 22.72.

2-(4-Chlorophenyl)-5-oxo-7-(pyridin-4-yl)-1,2,3,5-tetrahydro- $[1,2,4]$ triazolo $[1,5-a]$ pyridine- 6,8 -dicarbonitrile (11c)

Brown crystals; yield: $69 \%$; m.p. $>300{ }^{\circ} \mathrm{C}$; IR $(\mathrm{KBr})$ : $v_{\max } 3239,3297(2 \times \mathrm{NH}), 2217,2199(2 \times \mathrm{CN}), 1665(\mathrm{C}=\mathrm{O})$ $\mathrm{cm}^{-1} ;{ }^{1} \mathrm{H}$ NMR $\left(400 \mathrm{MHz}, \mathrm{DMSO}-d_{6}\right): \delta 4.85(\mathrm{~s}, 1 \mathrm{H}, \mathrm{NH})$, $5.15(\mathrm{~s}, 1 \mathrm{H}, \mathrm{CH}), 7.25(\mathrm{~s}, 1 \mathrm{H}, \mathrm{NH}), 7.31(\mathrm{~d}, J=8.2 \mathrm{~Hz}, 2 \mathrm{H}$, Ar-H), 7.41 (d, J=8.0 Hz, 2H, Ar-H), $7.58(\mathrm{~d}, J=7.1 \mathrm{~Hz}$, $2 \mathrm{H}, \mathrm{C}_{3}-\mathrm{H}, \mathrm{C}_{5}-\mathrm{H}$ pyridine), $8.66\left(\mathrm{~d}, J=7.2 \mathrm{~Hz}, 2 \mathrm{H}, \mathrm{C}_{2}-\mathrm{H}\right.$, $\mathrm{C}_{6}-\mathrm{H}$ pyridine); ${ }^{13} \mathrm{C}$ NMR $\left(100 \mathrm{MHz}\right.$, DMSO- $\left.d_{6}\right): \delta 87.63$, $100.06,116.24,116.88,122.64,122.89,128.64,129.33$, $134.16,143.08,144.73,150.24,151.24,162.45,171.22$; EIMS: $m / z 376\left(\mathrm{M}^{+}+2,0.88\right), 374\left(\mathrm{M}^{+}, 3 \%\right)$. Anal. Calcd. for $\mathrm{C}_{19} \mathrm{H}_{11} \mathrm{C}_{\mathrm{l}} \mathrm{N}_{6} \mathrm{O}$ (374.79): C, 60.89; H, 2.96; Cl, 9.46; N, 22.42 . Found: C, 60.93; H, 2.89; Cl, 9.51; N, 22.43.

Synthesis of 5-Oxo-7-(pyridin-4-yl)-3,5-dihydro-[1,2,4] triazolo[1,5-a]pyridine-6,8-dicarbonitrile (12)

A mixture of $9 \mathrm{a}(2.25 \mathrm{~g}, 0.01 \mathrm{~mol})$ and DMF-DMA $(1.19 \mathrm{~g}, 0.01 \mathrm{~mol})$ was heated under reflux in $50 \mathrm{~mL}$ of dry xylene for $8 \mathrm{~h}$. The solid product that precipitated on cooling was filtered off, dried and recrystallized from absolute ethanol to afford compound $\mathbf{1 2 .}$

Brown crystals; yield: 70\%; m.p. > $300{ }^{\circ} \mathrm{C}$; IR $(\mathrm{KBr})$ : $v_{\max } 3345(\mathrm{NH}), 2217,2227(2 \times \mathrm{CN}), 1684(\mathrm{C}=\mathrm{O}) \mathrm{cm}^{-1}$; ${ }^{1} \mathrm{H}$ NMR (400 MHz, DMSO- $\left.d_{6}\right): \delta 5.21(\mathrm{~s}, 1 \mathrm{H}, \mathrm{NH}), 8.01$ (d, J $=7.2 \mathrm{~Hz}, 2 \mathrm{H}, \mathrm{C}_{3}-\mathrm{H}, \mathrm{C}_{5}-\mathrm{H}$ pyridine), $8.79(\mathrm{~s}, 1 \mathrm{H}, \mathrm{CH})$, $8.98\left(\mathrm{~d}, J=7.2 \mathrm{~Hz}, 2 \mathrm{H}, \mathrm{C}_{2}-\mathrm{H}, \mathrm{C}_{6}-\mathrm{H}\right.$ pyridine); ${ }^{13} \mathrm{C} \mathrm{NMR}$ $\left(100 \mathrm{MHz}, \mathrm{DMSO}-d_{6}\right): \delta 115.27,115.98,117.54,122.49$, 123.08, 144.37, 149.88, 151.46, 155.21, 161.44, 171.83; EIMS: $m / z 262\left(\mathrm{M}^{+}, 10 \%\right)$. Anal. Calcd. for $\left(\mathrm{C}_{13} \mathrm{H}_{6} \mathrm{~N}_{6} \mathrm{O}\right)$ (262.23): C, 59.54; H, 2.31; N, 32.05. Found: C, 59.56; H, 2.27; N, 32.10 .

\section{3. Determination of the Anticancer Activity}

It was carried out according to the previously reported work. ${ }^{17}$

\section{Results and Discussion}

\section{1. Chemistry}

(E)-2-Cyano- $N$ '-(pyridin-4-ylmethylene)acetohydrazide (1) was synthesized by the reaction of isonicotinaldehyde with 2-cyanoacetohydrazide under various 


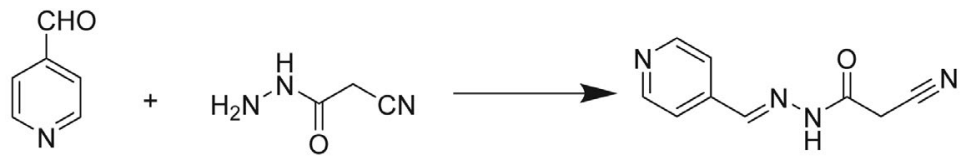

(1)

Scheme 1. Synthesis of (E)-2-cyano-N'-(pyridin-4-ylmethylene)acetohydrazide (1)

Table 1. Comparison of different conditions for the synthesis of $(E)$ 2-cyano- $N$ '-(pyridin-4-ylmethylene)acetohydrazide (1)

\begin{tabular}{cclcc}
\hline Entry & Catalyst & Condition & Time (h) & Yield (\%) \\
\hline 1 & No & Solvent free $\left(100^{\circ} \mathrm{C}\right)$ & 6 & 0 \\
2 & No & EtOH & 24 & 0 \\
3 & No & EtOH (reflux) & 9 & 28 \\
4 & TEA & EtOH (rt) & 24 & 0 \\
5 & TEA & EtOH (reflux) & 6 & 39 \\
6 & TEA & THF (reflux) & 4 & 92 \\
7 & No & Solvent free (MW) & $20 \mathrm{sec}$ & 98 \\
\hline
\end{tabular}

conditions (Scheme 1), and the results are presented in Table 1.

In the absence of any catalyst and under solvent-free conditons or in the presence of triethylamine as the basic catalyst at room temperature the reaction did not proceed even after long reaction time (Table 1, entries 1, 2 and 4). However, in the presence of $\mathrm{Et}_{3} \mathrm{~N}$ under reflux with the $\mathrm{EtOH}$ or THF as the solvents, the desired product was obtained in 39 or $92 \%$ yield, respectively (Table 1 , entries 5 and 6). Moreover, when the synthesis of $\mathbf{1}$ was carried out under microwave irradiation under solvent-free conditions, afforded the desired reaction product in high yield (Table 1, entry 7). The solvent-free conditions are preferrable as they avoid the use of toxic, flammable, and expen- sive organic solvents. The main advantages of microwave irradiation synthesis are thus shorter reaction time, higher yield and better purity of the product.

The chemical structure of $\mathbf{1}$ was confirmed by its spectral and elemental analysis data. The IR spectrum of $\mathbf{1}$ showed the presence of three stretching frequencies at 3235,2259 and $1704 \mathrm{~cm}^{-1}$ attributable to $\mathrm{NH}, \mathrm{CN}$ and $\mathrm{C}=\mathrm{O}$ groups, respectively. The ${ }^{1} \mathrm{H}$ NMR exhibited two singlet signals at $\delta 4.26$ and $8.25 \mathrm{ppm}$ due to $\mathrm{CH}_{2}$ and $\mathrm{CH}=\mathrm{N}$, respectively. In addition, two doublet signals at $\delta 8.02$ and $8.74 \mathrm{ppm}$ due to pyridine protons are observed. The configuration around the double bond of the compound 1 could not be established by ${ }^{1} \mathrm{H}$ NMR spectroscopy. However, the steric effect enhances that the $E$ isomer is more stable than $Z$ isomer.

Compound $\mathbf{1}$ acts as an adaptable material for the synthesis of novel pyridine compounds. Thus, refluxing of $\mathbf{1}$ and arylidene malononitriles $\mathbf{2 a - e}$ in ethanol catalyzed by piperdine afforded (E)-2-amino-4-aryl-5-cyano-6-oxo1-((pyridin-4-ylmethylene)amino)-1,6-dihydropyridine-3-carbonitriles $\mathbf{5 a}-\mathbf{e}$ (Scheme 2).

Formation of compounds $\mathbf{5 a}-\mathbf{e}$ could be elucidated by the mechanism presented in Scheme 2. At first, Michael addition of $\mathbf{1}$ to $\alpha, \beta$-unsaturated nitriles $\mathbf{2 a}-\mathbf{e}$ gives the intermediates $\mathbf{3}$. Then, the intermediates $\mathbf{3}$ undergo an interamolecular nucleophilic addition of $\mathrm{NH}$ group to the cyano function to afford the intermediates $\mathbf{4}$ and finally an<smiles>N#CCC(=O)N/N=C/c1ccncc1</smiles>

(1)<smiles>CCOC(=O)C=C(C#N)C(C)C</smiles>

(2)<smiles>N#CC(C#N)C(Br)C(C#N)C(=O)N/N=C/c1ccncc1</smiles><smiles>N#CC1C(=N)N(/N=C/c2ccncc2)C(=O)C(C#N)C1Br</smiles>

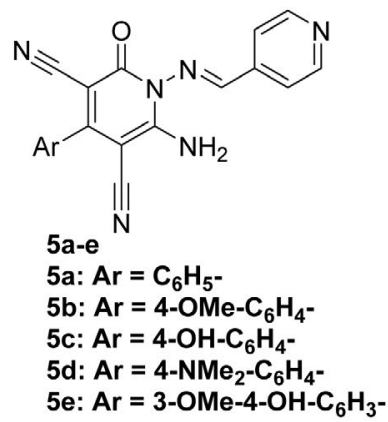

Scheme 2. Synthesis of (E)-2-amino-4-aryl-5-cyano-6-oxo-1-((pyridin-4-ylmethylene)amino)-1,6-dihydropyridine-3-carbonitriles 5a-e 


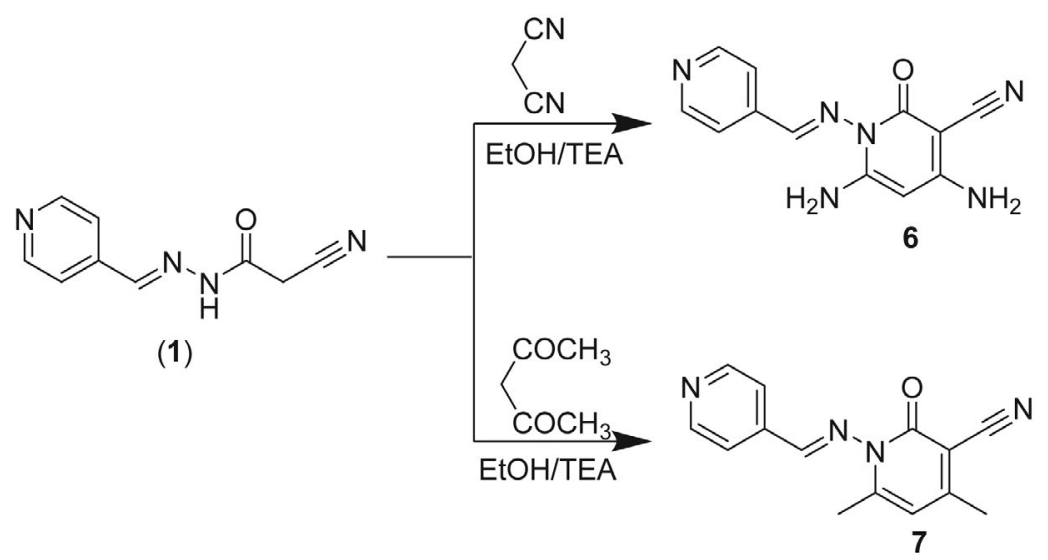

Scheme 3. Synthesis of (E)-2-oxo-1-((pyridin-4-ylmethylene)amino)-1,2-dihydropyridine-3-carbonitrile derivatives 6 and 7

autoxidation and tautomerization occur to give isolable products $5 \mathbf{a}-\mathbf{e} .{ }^{18}{ }^{1} \mathrm{H}$ NMR spectra of compounds $\mathbf{5 a}-\mathbf{e}$ display characteristic signals: singlet signal at $\delta 8.40-8.44$ ppm and two doublet signals at $\delta 7.99-8.04$ and 8.72-8.75 $\mathrm{ppm}$ due to the $\mathrm{CH}=\mathrm{N}$ and pyridine protons, respectively. Also, ${ }^{13} \mathrm{C}$ NMR revealed two signals in the region of $\delta$ $115-118 \mathrm{ppm}$ due to the two cyano groups in addition to the signal in the region of $\delta 161-163 \mathrm{ppm}$ attributable to the $\mathrm{C}=\mathrm{O}$ group. IR spectra of compounds $\mathbf{5 a - e}$ exhibited $\mathrm{NH}_{2}$ group stretching frequencies in the region of 3308$3397 \mathrm{~cm}^{-1}$ and the stretching frequency at 2200-2250 $\mathrm{cm}^{-1}$ that indicated the presence of two nitrile functional groups.

Treatment of $\mathbf{1}$ with malononitrile or acetylacetone in refluxing ethanol in the presence of trimethylamine as the base catalyst furnished (E)-4,6-diamino-2-oxo-1-((pyridin-4-ylmethylene)amino)-1,2-dihydropyridine-3-carbonitrile (6) and (E)-4,6-dimethyl-2-oxo-1-((pyridin-4-ylmethylene)amino)-1,2-dihydropyridine-3-carbonitrile (7), respectively (Scheme 3 ).

The spectral and analytical data of compounds 6 and 7 were in agreement with their proposed structures. ${ }^{1} \mathrm{H}$ NMR spectrum of $\mathbf{6}$ showed two singlet signals at $\delta 4.62$ and $8.43 \mathrm{ppm}$ owing to the $\mathrm{C}_{5}-\mathrm{H}$ of 2 -pyridone ring and $\mathrm{CH}=\mathrm{N}$, respectively. Moreover, ${ }^{1} \mathrm{H}$ NMR of $\mathbf{6}$ exhibited two singlet signals $\left(\mathrm{D}_{2} \mathrm{O}\right.$-exchangable) at $\delta 4.65$ and 6.68 ppm due to the two $\mathrm{NH}_{2}$ groups. The IR analysis substantiated the results of ${ }^{1} \mathrm{H}$ NMR by the presence of four peaks in the region of $3321-4000 \mathrm{~cm}^{-1}$ for two $\mathrm{NH}_{2}$ groups.

Nowadays, multicomponent reactions are gaining extensive economic and ecological importance as they conform to the fundamental principles of synthetic efficiency and reaction design. ${ }^{19}$ We herein provide an efficient and facile procedure for the synthesis of 4,4'-bipyridine derivatives 9a-d via a one-pot three-components condensation of isonicotinaldehyde, 2-cyanoacetohydrazide and activated nitriles 8a- $\mathbf{d}^{20,21}$ (Scheme 4).

The structures of products $9 \mathbf{a}-\mathbf{d}$ were assigned according to their IR, ${ }^{1} \mathrm{H}$ NMR, ${ }^{13} \mathrm{C}$ NMR and mass spectra. Thus, all compounds $\mathbf{9 a}-\mathbf{d}$ gave molecular ion peak which coincides with the proposed structure. ${ }^{1} \mathrm{H}$ NMR gave an additional evidence for the correct structure of compounds $\mathbf{9 a - d}$, for example, compound $\mathbf{9 c}$ gave triplet quartet pattern at $\delta 1.01$ and $4.03 \mathrm{ppm}$, respectively, which confirm the presence of ethyl ester group in addition to the singlet signal $\left(\mathrm{D}_{2} \mathrm{O}\right.$-exchangable $)$ at $\delta 7.08 \mathrm{ppm}$ corresponding to the amino group. Compound $9 \mathbf{a}$ was used as a versatile material for the synthesis of 2-aryl-5-oxo-7-(pyridin-4-yl)-1,2,3,5-tetrahydro-[1,2,4] triazolo[1,5-a]pyridine-6,8-dicarbonitrile derivatives 11a-c. Consequently, the reaction of $\mathbf{9 a}$ with aromatic aldehydes $\mathbf{1 0} \mathbf{a}-\mathbf{c}$ afforded compounds 11a-c (Scheme 5).<smiles>N#CCC(=O)NN</smiles><smiles>[R]c1c(-c2ccncc2)c(C#N)c(=O)n(N)c1N</smiles>

$$
\begin{aligned}
& \text { 9a-d } \\
& \text { 9a: } \mathrm{R}=\mathrm{CN} \\
& \text { 9b: } \mathrm{R}=\mathrm{SO}_{2} \mathrm{Ph} \\
& \text { 9c: } \mathrm{R}=\mathrm{COOEt} \\
& \text { 9d: } \mathrm{R}=\text { 2-benzothiazolyl }
\end{aligned}
$$

Scheme 4. Synthesis of 5-alkyl-1,6-diamino-2-oxo-1,2-dihydro-[4,4'-bipyridine]-3-carbonitriles 9a-d 
<smiles>N#Cc1c(-c2ccncc2)c(C#N)c(=O)n(N)c1N</smiles>

9a

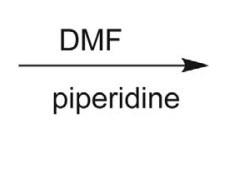<smiles>N#Cc1c(-c2ccncc2)c(C#N)c(=O)n2c1NC([Al])N2</smiles>

11a-c

11a: $\mathrm{Ar}=\mathrm{C}_{6} \mathrm{H}_{5}$ 11b: $\mathrm{Ar}=4-\mathrm{OMe}-\mathrm{C}_{6} \mathrm{H}_{4}-$ 11c: $\mathrm{Ar}=4-\mathrm{Cl}-\mathrm{C}_{6} \mathrm{H}_{4}-$

Scheme 5. Synthesis of 2-aryl-5-oxo-7-(pyridin-4-yl)-1,2,3,5-tetrahydro-[1,2,4]triazolo[1,5-a]pyridine-6,8-dicarbonitriles 11a-c<smiles>N#Cc1c(-c2ccncc2)c(C#N)c(=O)n(N)c1N</smiles>

9a

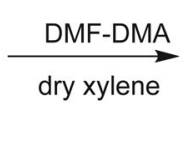<smiles>N#Cc1c(-c2ccncc2)c(C#N)c2[nH]cnn2c1=O</smiles>

12
Scheme 6. Synthesis of 5-oxo-7-(pyridin-4-yl)-1,5-dihydro-[1,2,4] triazolo[1,5-a]pyridine-6,8-dicarbonitrile (12)

Structures 11a-c were established on the basis of elemental analyses and spectral data. The ${ }^{1} \mathrm{H}$ NMR spectra of compounds 11a-c, in general, gave singlet signal at $5.08-5.15 \mathrm{ppm}$ attributable to $\mathrm{C}_{3}-\mathrm{H}$ of $[1,2,4]$ triazole ring in addition to the two singlet signals $\left(\mathrm{D}_{2} \mathrm{O}\right.$-exchangable) at $\delta 4.77-4.85$ and $7.25-7.29 \mathrm{ppm}$ due to the two $\mathrm{NH}$ groups.

Also, 5-oxo-7-(pyridin-4-yl)-1,5-dihydro-[ $[1,2,4]$ triazolo[1,5-a]pyridine-6,8-dicarbonitrile (12) was synthesized via cyclocondensation reaction of $\mathbf{9 a}$ with DMFDMA (Scheme 6).

\section{2. Pharmacology}

\section{2. 1. Cytotoxicity Against Hepatoma Cell Line (HepG-2) and Human Breast Adenocarcinoma Cell Line (MCF-7)}

Cytotoxic activity. In order to investigate if the chemistry established here has led to possibly interesting nominees in cancer therapy, our primary aim was directed towards checking if the novel synthesized compounds possess any anticancer activities as predicted by this study. In vitro cytotoxic study was therefore performed against two mammalian cancer cell lines, HepG-2 (hepatoma cells or human liver hepatocellular carcinoma cell line) and MCF-7 (human breast adenocarcinoma cell line). This study indicated that compounds $\mathbf{9 a}$ and $\mathbf{9 b}$ showed very strong cytotoxic activity against HepG-2 cancer cells with $\mathrm{IC}_{50}$ values of $8.83 \pm 0.30$ and $10.08 \pm 0.66 \mu \mathrm{g} / \mathrm{mL}$, respectively. Also, both $\mathbf{9 a}$ and $\mathbf{9 b}$ gave high cytotoxic effects against MCF-7 classifying these compounds as chemotherapeutically significant (Table 2). The rest of other compounds showed a moderate to weak activity against the tested tumor cell lines. $\mathrm{IC}_{50}$ is the concentration, which can reduce the growth of cancer cells by $50 \%$.
Table 2. Cytotoxic activity of the newly synthesized compounds

\begin{tabular}{|c|c|c|}
\hline \multirow[t]{2}{*}{ Compounds } & \multicolumn{2}{|c|}{ In vitro Cytotoxicity $\mathrm{IC}_{50}(\mu \mathrm{g} / \mathrm{mL})$} \\
\hline & HepG-2 & MCF-7 \\
\hline 5-FU & $7.53 \pm 0.22$ & $4.05 \pm 0.15$ \\
\hline 1 & $45.83 \pm 0.28$ & $37.66 \pm 0.35$ \\
\hline $5 \mathbf{a}$ & $78.53 \pm 1.25$ & $84.20 \pm 2.23$ \\
\hline $5 \mathbf{b}$ & $31.11 \pm 0.38$ & $22.27 \pm 0.33$ \\
\hline $5 c$ & $18.81 \pm 0.14$ & $16.08 \pm 0.28$ \\
\hline $5 d$ & $20.23 \pm 0.21$ & $31.55 \pm 0.34$ \\
\hline $5 e$ & $16.97 \pm 0.27$ & $14.99 \pm 0.31$ \\
\hline 6 & $>100$ & $>100$ \\
\hline 7 & $52.65 \pm 0.68$ & $64.37 \pm 1.20$ \\
\hline 9a & $8.83 \pm 0.30$ & $10.37 \pm 0.24$ \\
\hline $9 b$ & $10.08 \pm 0.66$ & $8.98 \pm 0.61$ \\
\hline $9 c$ & $89.34 \pm 1.33$ & $64.38 \pm 1.08$ \\
\hline 9d & $71.08 \pm 0.98$ & $62.35 \pm 0.55$ \\
\hline $11 a$ & $69.35 \pm 1.30$ & $58.98 \pm 0.84$ \\
\hline $11 b$ & $43.55 \pm 0.68$ & $39.63 \pm 0.50$ \\
\hline $11 \mathrm{c}$ & $>100$ & $>100$ \\
\hline 12 & $94.64 \pm 1.33$ & $80.37 \pm 1.58$ \\
\hline
\end{tabular}

$\mathrm{IC}_{50}(\mu \mathrm{g} / \mathrm{mL})$ : $1-10$ very strong, $11-20$ strong, $21-50$ moderate, 51-100 weak and above 100 non-cytotoxic. 5-FU is 5-fluorouracil.

\section{Conclusion}

In conclusion, herein we report a simple and convenient method for the synthesis of novel pyridine derivatives. All synthesized compounds were evaluated against two cancer cell lines (HepG-2 and MCF-7). Among all the synthesized compounds, compounds $\mathbf{9 a , b}$ have high cytotoxic activity against both HepG-2 and MCF-7. The rest of compounds showed a moderate to weak activity against the tested tumor cell lines.

\section{References}

1. (a) G. Jones, Comprehensive Heterocyclic Chemistry II, Vol. 5 (Eds.: A. R. Katritzky, C. W. Rees, E. F. V. Scriven, A. McKillop), Pergamon, Oxford, 1996, pp. 167-243;

DOI:10.1016/B978-008096518-5.00108-8

(b) G. D. Henry, Tetrahedron 2004, 60, 6043-6061.;

DOI:10.1016/j.tet.2004.04.043 
(c) J. A. Joule, K. Mills, Heterocyclic Chemistry, $4^{\text {th }}$ ed., Blackwell Science, Cambridge, 2000; p. 63-120;

(d) J. P. Michael, Nat. Prod. Rep. 2005, 22, 627-646. DOI:10.1039/b413750g

2. M. T. Cocco, C. Congiu, V. Lilliu, V. Onnis, Eur. J. Med. Chem. 2005, 40, 1365-1372. DOI:10.1016/j.ejmech.2005.07.005

3. D. L. Boger, S. Nakahara, J. Org. Chem. 1991, 56, 880-884. DOI:10.1021/jo00002a077

4. D. L. Boger, A. M. Kasper, J. Am. Chem. Soc. 1989, 111, 15171519. DOI:10.1021/ja00186a067

5. T. S. Harrison, L. J. Scott, Drugs 2005, 65, 2309-2336. DOI:10.2165/00003495-200565160-00010

6. M. W. Deininger, B. J. Druker, Pharmacol. Rev. 2003, 55, 401423. DOI:10.1124/pr.55.3.4

7. E. V. Costa, M. L. B. Pinheiro, C. M. Xavier, J. R. Silva, A. C. F. Amaral, A. D. Souza, A. Barison, F. R. Campos, A. G. Ferreira, G. M. A. Machado, J. Nat. Prod. 2006, 69, 292-294. DOI:10.1021/np050422s

8. R. H. Bahekar, M. R. Jain, P. A. Jadav, V. M. Prajapati, D. N. Patel, A. A. Gupta, A. Sharma, R. Tom, D. Bandyopadhya, H. Modi, Bioorg. Med. Chem. 2007, 15, 6782-6795.

DOI:10.1016/j.bmc.2007.08.005

9. F. Shi, C. Li, M. Xia, K. Miao, Y. Zhao, S. Tu, W. Zheng, G. Zhang, N. Ma, Bioorg. Med. Chem. Lett. 2009, 19, 5565-5568. DOI:10.1016/j.bmcl.2009.08.046

10. K. Nicolaou, R. Scarpelli, B. Bollbuck, B. Werschkun, M. Pereira, M. Wartmann, K. Altmann, D. Zaharevitz, R. Gussio, P. Giannakakou, Chem. Biol. 2000, 7, 593-599.

DOI:10.1016/S1074-5521(00)00006-5
11. J.-P. Liou, K.-S. Hsu, C.-C. Kuo, C.-Y. Chang, J.-Y. Chang, J. Pharmacol. Exp. Ther. 2007, 398-405.

DOI:10.1124/jpet.107.126680

12. R. M. Mohareb, N. Y. M. Abdo, F. O. Al-Farouk, Acta Chim. Slov. 2017, 64, 117-128. DOI:10.17344/acsi.2016.2920

13. J. M. Chezal, J. Paeshuyse, V. Gaumet, D. Canitrot, A. Maisonial, C. Lartigue, A. Gueiffier, E. Moreau, J. C. Teulade, O. Chavignon, Eur. J. Med. Chem. 2010, 45, 2044-2047. DOI:10.1016/j.ejmech.2010.01.023

14. W. Xie, Y. Wu, J. Zhang, Q. Mei, Y. Zhang, N. Zhu, R. Liu, H. Zhang, Eur. J. Med. Chem. 2018, 145, 35-40.

DOI:10.1016/j.ejmech.2017.12.038

15. N. K. Binsaleh, C. A. Wigley, K. A. Whitehead, M. van Rensburg, J. Reynisson, L. I. Pilkington, D. Barker, S. Jones, N. C. Dempsey-Hibbert, Eur. J. Med. Chem. 2018, 143, 1997-2004. DOI:10.1016/j.ejmech.2017.11.014

16. Q. Tang, Y. Duan, L. Wang, M. Wang, Y. Ouyang, C. Wang, H. Mei, S. Tang, Y. Xiong, P. Zheng, P. Gong, Eur. J. Med. Chem. 2018, 143, 266-275. DOI:10.1016/j.ejmech.2017.11.034

17. A. A. Fadda, E. Abdel-Latif, R. E. El-Mekawy, Pharmacol Pharm 2012, 3, 148-157. DOI:10.4236/pp.2012.32022

18. M. R. H. Elmoghayar, A. G. A. El-Agamey, M. Y. A. S. Nasr, M.m. M. Sallam, J. Het. Chem. 1984, 21, 1885-1887.

19. C. C. Cariou, G. J. Clarkson, M. Shipman, J. Org. Chem. 2008, 73, 9762-9764. DOI:10.1021/jo801664g

20. A. H. M. Hussein, Heteroat. Chem. 1997, 8, 1-6. DOI:10.1002/(SICI)1098-1071(1997)8:1<1::AID-HC1>3.0. CO; 2-J

21. F. F. A. Latif, R. Mekheimer, E. K. Ahmed, T. B. A. Aleem, Pharmazie 1993, 48, 736-738.

\section{Povzetek}

Reakcija izonikotinaldehida z 2-cianoacetohidrazidom daje (E)-2-ciano-N'-(piridin-4-ilmetilen)acetohidrazid (1). Spojino 1 smo uporabili kot prekurzor pri sintezi novih piridinskih derivatov, ki smo jih pripravili z reakcijo med različnimi arilidenskimi malononitrili, malononitrilom oz. acetilacetonom, pri čemer so nastali piridinski derivati 5a-e, 6 in 7 . 4,4'-Bipiridinske derivate 9a-d smo pripravili s trokomponentno reakcijo med izonikotinaldehidom, 2-cianoacetohidrazidom in aktiviranimi nitrili 8a-d. Obdelava spojine 9a z različnimi aromatskimi aldehidi je vodila do nastanka $[1,2,4]$ triazolo[1,5-a]piridinskih derivatov $\mathbf{1 1 a}-\mathbf{c}$. Vse reakcijske produkte smo okarakterizirali $\mathrm{z}$ analitskimi in spektroskopskimi metodami. Za nove spojine smo raziskali tudi bioaktivnost $\mathrm{v}$ vlogi antitumornih učinkovin $\mathrm{v}$ in vitro testiranju citotoksičnosti proti HepG-2 in MCF-7. Ugotovili smo, da spojini 9a in $9 \mathbf{b}$ izkazujeta visoko citotoksičnost proti obema, HegG-2 in MCF-7. 